\title{
Decoherence due to thermal effects in two quintessential quantum systems
}

\author{
S. Nussinov $\cdot$ T. Madziwa-Nussinov $\cdot$ Z. Nussinov
}

Received: 22 April 2014 / Accepted: 27 April 2014 / Published online: 11 June 2014

(C) Chapman University 2014

\begin{abstract}
Decoherence effects at finite temperature ( $T$ ) are examined for two manifestly quantum systems: (i) Casimir forces between parallel plates that conduct along different directions, and (ii) a topological AharonovBohm (AB) type force between fluxons in a superconductor. As we illustrate, standard path integral calculations suggest that thermal effects may remove the angular dependence of the Casimir force in case (i) with a decoherence time set by $h /\left(k_{B} T\right)$ where $h$ is Plank's constant. This prediction may be tested. The effect in case (ii) is due to a $\pi$ phase shift picked up by unpaired electrons upon encircling an odd number of fluxons. In principle, this effect may lead to small modifications in Abrikosov lattices. While the AB forces exist at extremely low temperatures, we find that at the pertinent temperatures $T=\mathcal{O}\left(T_{c}\right)$ (the critical temperature of the superconductor), thermal decoherence may strongly suppress the topological force. It is suggested that both cases (i) and (ii) (as well as other examples briefly sketched) are related to a quantum version of the fluctuation-dissipation theorem.
\end{abstract}

Keywords Quantum decoherence · Casimir forces · Topological forces · Superconductivity · Fluctuation dissipation

\section{Introduction}

A quantum system in equilibrium at a finite temperature is not in a pure state, but rather is described by a thermal density matrix. However, just like a classical pendulum or a loaded spring which keeps performing for some time their periodic motion in a thermal environment, so can an appropriately entangled quantum system keep its coherence for a while. The thermal environment generates a random fluctuating force operating on the classical harmonic oscillator system. The "fluctuation-dissipation theorem" (see, e.g., [1]) then implies that these damp the

\footnotetext{
S. Nussinov

Raymond and Beverly Sackler School of Physics and Astronomy, Tel Aviv University, Tel Aviv, Israel

S. Nussinov

Institute for Quantum Studies, Chapman University, Orange, CA 92866 , USA

T. Madziwa-Nussinov $\cdot$ Z. Nussinov $(\bowtie)$

Physics Department, Washington University, St. Louis, MO 63130 , USA

e-mail: zohar@wuphys.wustl.edu
} 
initial ordered periodic motion leaving only the $O\left(k_{B} T\right)$ thermal energy per degree of freedom. Similarly, thermal effects will tend to decohere the quantum system, which started say as a pure state [2].

Rather than addressing the full and involved question of the general quantum version of the fluctuation-dissipation theorem, we address here two examples. These are two effects which to certain degrees are spoiled and weakened by a randomizing and decohering thermal milieu. While these effects seem superficially to be unrelated, we believe that at a deeper level they are and both pertain to the general issue considered.

The first effect is a "polarized" version of the Casimir force between two neutral parallel conducting plates [3]. It arises when the two plates conduct in different directions. Beyond its $1 / a^{4}$ dependence on the separation $a$ of the two plates, the Casimir force per unit of area then depends also on the angle $\beta$ between the directions of conductance [4]. This dependence reflects the vector nature of light, namely, the polarization degree of freedom. The Casimir force and its variant can be viewed, like many other forces, as being generated by the interaction of the plates (or "big" objects in general) with smaller elements or modes of the intervening medium. The forces can be calculated exactly using the euclidean path integral method introduced and extensively used in this context in the thesis of Kenneth [5].

The Casimir force per unit area at finite temperatures is well known [6] and can be readily derived by the path integral approach where the time direction is compactified down to a size of $1 /\left(k_{B} T\right)$. When $k_{B} T>1 / a$, the thermal Casimir force has a $k_{B} T / a^{3}$ dependence and is stronger than the ordinary (i.e., zero temperature) Casimir force-as indeed suggested by a simplistic argument.

Below, we evaluate the polarized variant of the Casimir effect for finite temperature. This calculation was motivated by the prospect that the effect will be measured experimentally [7]. The setup considered was at room temperature of $300 \mathrm{~K}$, i.e., $k_{B} T \sim e V / 40$ and plate separation a of few microns. Contrary to "naive expectations", the calculation implies that the dependence of the force on $\beta$, the angle between the conduction directions of the plate, falls off exponentially with temperature like $\exp \left(-2 \pi k_{B} T a /(\hbar c)\right)$. This is attributed to the randomizing effect on the polarization of the photons in a thermal background as they travel between the two plates.

The second effect concerns the "Aharonov-Bohm Force" suggested to occur [8] between magnetic fluxons immersed in a fluid of electrons due to phases which the electrons pick when circulating the fluxons. In particular, for "semions", namely half-fluxons-in units of $h c / e$ where $e$ is the electron rather than the Cooper pair charge of $2 e$-which naturally arise inside superconductors, this 'topological' force was found to be attractive even for fluxons with parallel magnetic fields. This was inspired on the one hand by analogy with the Casimir effect with the electrons (rather than the vacuum and/or thermal photons) playing the role of the relevant excitations of the medium and by some remarkable features of the the ground state of electronic system in the presence of half-fluxons [9]. To achieve it in a real setup of physical superconductors, we have to use a finite temperature $T<T_{c}$ so that we have both superconductivity and fluxons and a finite fraction of unpaired electrons to generate the force of the form $F \sim n \hbar^{2} / m_{e} r$ with $r$ the separation between the fluxons and $n$ the two-dimensional number density of the electrons. In Ref. [8], it was remarked that unless somehow screened by countering currents, the force was rather large.

Here, we note that decohering effects can randomize the electrons' phases over time intervals of order $1 / k_{B} T$ and naturally lead to an exponentially falling force between two nearby semions. The reduced symmetry of the present problem relative to the case of the Casimir parallel infinite plates, hinders exact evaluation of the thermal path integral though it suggests the same qualitative results. We next speculate on the feasibility of testing the effect now that we have it in a more realistic and correct form.

The plan of the paper is as follows: Section 2 presents a discussion of the rather long relaxation time of a simple classical harmonic motion in certain cases even in the presence of a thermal background. In Sects. 3 and 4, we briefly review the two effects/forces mentioned above and present the new and more involved finite temperature discussion. In Sect. 5, we comment on the connection with the general theme of thermal decoherence and conclude. 


\section{A short note on classical decoherence}

It is instructive to recall how classical systems can maintain "coherent" motion despite a thermal background—as we conjecture that similar persistence effects may be relevant also to the quantum case. Consider then an 'ideal' sensitive torsion balance designed to measure tiny—say, gravitational forces. It consists of a horizontal bar hung at its center by a thin long and ideally (!!) a loss-free fiber. The system is enclosed inside a cylinder pumped to very low pressure of $\sim 10^{-10}$ atmospheres. When gravitationally torqued by a pair of massive near-by spheres, the bar and fiber system starts performing a periodic harmonic twist motion.

The question of interest is the extent to which finite, say even room temperature, effects of the medium impair the function of the torsion balance. For generic $O(0.1) \mathrm{kg}$ masses of rod and external spheres, dimensions and distances of $O(10) \mathrm{cm}$, and a nano-radian rotation of the bar, the gravitational energy involved and that of the resulting energy in the oscillatory mode are comparable with or smaller than $k_{B} T$, the generic thermal energy per degree of freedom. However, only after that much energy is pumped to that particular mode of motion of the rod will its motion be appreciably affected. After the system relaxes to thermal equilibrium - all degrees of freedom and that of the whole rod considered here as well, will have, by the equipartition theorem, each have an energy of $k_{B} T$. However, if this relaxation is driven only by collisions with the ambient residual gas molecules (or with thermal photons which only drastic cooling can eliminate), the times required for damping this motion are very long and hence this effect does not hinder the performance of useful experiments. ${ }^{1}$

\section{The "polarized"-conducting direction-dependent Casimir effect at finite temperatures}

\subsection{A path integral approach}

In the standard case of isotropic conducting plates of area $A$, the Casimir force per unit area [6] is given by

$$
\frac{F_{\text {cas }}}{A}=-\frac{\pi^{2}}{240} \frac{\hbar c}{a^{4}}
$$

We now consider the case of plates which conduct only along one direction.

The exact evaluation of the Casimir energy/force per unit area utilizes the path integral. For clarity, we reproduce some of key steps from an earlier work [4]. Unless explicitly inserted otherwise, we will set both the speed of light $c$ and the Boltzmann constant $k_{B}$ to unity $\left(c=k_{B}=1\right)$ in the calculations that follow. In the presence of the conducting parallel plates 1 and 2 , which are both parallel to the $x-y$ plane and separated by a distance " $a$ " along the $z$ axis the partition function is given by

$Z=\int D \mathcal{A} D J \exp \left(-i \int d^{4} x \frac{F^{2}}{4}+i \int d^{3} x \mathcal{A} \cdot J\right)$,

where $F_{\mu \nu}$ are the standard EM fields and $J$ denotes the currents. In this last term, the scalar product $\mathcal{A} \cdot J$ is performed only along the area-time of the plates. If both plates conduct only along a single spatial direction, then the currents $J=J_{1}+J_{2}$ will have spatial projection restricted to the $i$ th plate (along the specified conductance directions in each of the plates). These currents serve as Lagrange multipliers forcing the vanishing of the respective components of the electric fields along the two plates. Integrating (after a Wick rotation) the quadratic form in $\mathcal{A}_{\mu}$ we find

$$
\int D J \exp \left[-\int d^{3} x d^{3} y\left(\frac{J_{1}(x) \cdot J_{1}(y)+J_{2}(x) \cdot J_{2}(y)}{(x-y)^{2}}+\frac{2 J_{1}(x) \cdot J_{2}(y)}{(x-y)^{2}+a^{2}}\right)\right],
$$

where we used the conservation of $J_{1}$ and $J_{2}$ and the resulting gauge freedom to choose the simple configuration space Feynman propagator in 4-dimensional space-time, $\Delta_{F}^{\mu \nu}(x-y)=g^{\mu \nu} / 2 \pi^{2}(|x-y|)^{2}$. It is important to

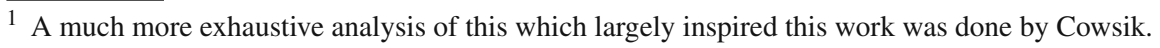


underscore that the currents $J_{1,2}$, the coordinates, and the momenta $k$ to appear shortly, all live in the 3-dimensional $(x, y, t)$ space. The first two terms in Eq. (3) refer to the "self interactions" of currents in the individual plates and the third to the mutual plate-plate interactions-hence the extra factor of $a^{2}$ in the denominator of the propagator, with a the plate spacings in the $z$ direction. Fourier transforming Eq. (3) which amounts to the (unitary) change of variable from $J(x)$ to $J(k)$-we find, thanks to the translation invariance of the propagator,

$\int D J(\mathbf{k}) \exp \left[-\int d^{3} k\left(\frac{J_{1}(\mathbf{k}) \cdot J_{1}(-\mathbf{k})+J_{2}(\mathbf{k}) \cdot J_{2}(-\mathbf{k})}{k}+\frac{2 J_{1}(\mathbf{k}) \cdot J_{2}(-\mathbf{k})}{k} e^{-k a}\right)\right]$.

The conservation $\partial^{\mu} J_{\mu}(x)=0$, or $k \cdot J(k)=0$, of the currents and the angle $\beta$ between their spatial $(x y)$ projections (along the conductance directions in the two plates) fixes the cosine of the angle between the (three dimensional) $J_{1}(k)$ and $J_{2}(k)$ which we denote by $\alpha(\hat{k})$ and $J_{1} \cdot J_{2}=J_{1} J_{2} \cdot \alpha(\hat{k})$. The integration of the quadratic (in $J_{i}(k)$ ) action produced the usual product over modes $k$ of the $2 \times 2$ determinants,

$Z=\prod_{\mathbf{k}} \operatorname{det}\left(\begin{array}{ll}\frac{1}{k} & \frac{(\alpha \hat{k})}{k} e^{-k a} \\ \frac{(\alpha \hat{k})}{k} e^{-k a} & \frac{1}{k}\end{array}\right)^{-\frac{1}{2}}$.

At zero temperature, taking the $\log$ of $Z$, transforming to the continuum limit in the large volume $=A T$ with $A$ the area of the plates and $T$ the long time duration, so as to replace the mode sum by an integral, and discarding an infinite constant that is independent of $a$ (plate separation) and $\beta$ (the angle between the conductance directions), we find that

$$
\begin{aligned}
\ln Z & =-\frac{1}{2} \sum_{\mathbf{k}} \ln \operatorname{det}(\ldots) \\
& =-\frac{1}{2} A T \int \frac{d^{3} k}{(2 \pi)^{3}} \ln \left(1-\alpha(\hat{k})^{2} e^{-2 k a}\right)+\text { const. }
\end{aligned}
$$

Using $E=(\ln Z) / T$, we finally obtain the Casimir energy per unit area

$\frac{E}{A}=\frac{1}{2} \int \frac{d^{3} k}{(2 \pi)^{3}} \ln \left[1-\alpha^{2}(\hat{k}) e^{-2 k a}\right]$.

The three vector $\mathbf{k}=k \hat{k}$ is, in polar coordinates,

$$
\begin{aligned}
\mathbf{k} & =\left(k_{x}, k_{y}, k_{z}\right)=\left(k_{x}, k_{y}, \omega\right) \\
& =k(\sin \theta \cos \varphi, \sin \theta \sin \varphi, \cos \theta) .
\end{aligned}
$$

This yields an explicit expression for $\alpha^{2}$ as in [4],

$$
\begin{aligned}
\alpha^{2} & =\left(\hat{J}_{1}(\mathbf{k}) \cdot \hat{J}_{2}(\mathbf{k})\right)^{2} \\
& =\frac{\left[\cos \beta-\sin ^{2} \theta \cos \varphi \cos (\varphi-\beta)\right]^{2}}{\left(1-\sin ^{2} \theta \cos ^{2} \varphi\right)\left(1-\sin ^{2} \theta \cos ^{2}(\varphi-\beta)\right)} .
\end{aligned}
$$

The last two equations are the starting point for the present discussion of the Casimir force dependence on the angle $\beta$ between the conductance directions for non-zero temperature T (not to be confused with the Euclidean time above). The latter manifests by replacing the energy, $\omega=k_{t}$ integration above by a sum over the Matsubara frequencies $\omega(n)=2 \pi n k_{B} T / \hbar \equiv n \tau$

$$
\int \frac{d k_{t}}{2 \pi} \rightarrow T \sum_{-\infty}^{\infty}[\text { Matsubara frequencies }] \text {. }
$$


Using $k=\left(k_{x}^{2}+k_{y}^{2}+\omega^{2}\right)^{1 / 2} \equiv\left(K^{2}+\omega^{2}\right)^{1 / 2}$, we then have the Casimir free energy per unit area at non-zero temperatures,

$$
\frac{E}{A}=\frac{T}{2} \int \frac{d^{2} K}{(2 \pi)^{2}} \sum_{n=-\infty}^{\infty} \ln \left[1-\alpha^{2}(\hat{K}) e^{-2 \sqrt{K^{2}+(n \tau)^{2}} a}\right] .
$$

The last equation and further analysis below imply that at high temperatures (or high lowest non-vanishing Matsubara frequency $n \tau)$, all $n \neq 0$ terms vanish exponentially. Since the only other dimensionful energy parameter in the problem is $1 / a$ with a the plate separation, the "high temperature" dimensionless parameter $\xi$ where $\xi=2 \pi T a=\omega_{1} a$ which is the ratio of the two energy scales must be large. The key observation is that if we keep only the lowest $\mathrm{n}=0$ mode we lose all dependence on the angle $\beta$ between the conductance directions!

As seen from Eq. (8), a Matsubara index $n=0$, or equivalently, $\omega=0$, implies that $\sin \theta=1, \cos \theta=0$. Substituting Eq. (9), we find that $\alpha^{2}=1$ identically and

$$
\left.\frac{E}{A}\right|_{n=0}=\frac{T}{2} \int \frac{K d K}{(2 \pi)^{2}} \int d \varphi \ln \left(1-e^{-2 K a}\right) .
$$

By expanding the integrand in powers of $\exp (-2 K a)$, integrating each term, and summing up the resulting series $\sum_{l=1}^{\infty}\left(1 / l^{3}\right)=\zeta(3) \approx 1.202$, we obtain the high temperature limit of the Casimir energy: $E / A \approx T / a^{2}$. The corresponding Casimir force $F / A=-\frac{d}{d a}(E(a, T))=$ const. $\left(T / a^{3}\right)$ is indeed motivated by heuristic arguments to be briefly presented later. At high temperatures $(\xi \gg 1)$, this "finite temperature Casimir force" is larger than that of the zero-temperature pure case of plates in the vacuum by a factor of order $\xi$. Our main concern here though is the resulting dramatic suppression of the dependence on the relative angle $\beta$ between the conductance direction in the two plates. The maximal variation of the Casimir energy as a function of $\beta$ is obtained as $\beta$ varies from $\beta=0$ to $\beta=\pi / 2$, namely that between parallel and perpendicular conductivities.

In the first, parallel, case we have from Eq. (9) that $\alpha=1$ identically yielding $E / A$,

$$
E /\left.A\right|_{(\beta=0, T)}=\frac{T}{2} \int \frac{d^{2} K}{(2 \pi)^{2}} \sum_{n=-\infty}^{\infty} \ln \left(1-e^{-2 \sqrt{K^{2}+(n \tau)^{2}} a}\right),
$$

at general temperature $T$.

The case of $\beta=\pi / 2$ is more involved. Denoting by $K$ the two- dimensional spatial vector in the $x, y$ plane, we have $\sin ^{2} \theta=K^{2} /\left(K^{2}+\omega^{2}\right)=K^{2} /\left(K^{2}+(n \tau)^{2}\right)$ and for $n \neq 0$

$\left.\alpha^{2}\right|_{\beta=\frac{\pi}{2}}=\frac{\left(K^{2} \cos \varphi \sin \varphi\right)^{2}}{\left[(n \tau)^{2}+K^{2} \sin ^{2} \varphi\right]\left[(n \tau)^{2}+K^{2} \cos ^{2} \varphi\right]}<1$.

This should be substituted in

$$
\frac{E}{A}\left(\beta=\frac{\pi}{2}, T\right)=\frac{T}{8 \pi^{2}} \int d \varphi \int K d K \sum_{n=-\infty}^{\infty} \ln \left[1-\alpha_{\left(\beta=\frac{\pi}{2}\right)}^{2} e^{-2 \sqrt{K^{2}+(n \tau)^{2}} a}\right] .
$$

It is difficult to separate the plates in the parallel planar geometry required here, by less than a micron, so that $a \geq 10^{-4} \mathrm{~cm}$. At room temperature, $T=300 \mathrm{~K}$, we find already for this small separation $a$ that the largest first, $n= \pm 1$ terms is small, i.e., with $c$ inserted, $2 \alpha_{\beta=\pi / 2}^{2} \exp \left(-2 \sqrt{K^{2}+(2 \pi a T)^{2}}\right)<2 \exp \left(-4 \pi k_{B} T a /(\hbar c)\right) \approx 0.353$ for $a=1$ micron.

The largest contribution to the Casimir free energy and force originates from the first $(n=0)$ term in the Matsubara sum which is angle $(\beta)$ independent $\left(\alpha(n=0)^{2}=1\right)$. The $\beta$ dependence originating from the non-zero 
Matsubara frequencies is reduced by a factor of three for a plate separation of a micron (and is exponentially decreasing in $a$ ). Reserving a more careful evaluation of this effect at various temperatures to future work, we still wish to have a better physical understanding as to why the rather strong $\beta$ dependence at $T=0$ disappears so fast at finite $T$. To this end, we briefly review heuristic albeit crude arguments that seem to suggest that the $\beta$ dependence is not altogether removed.

\subsection{The heuristic approach, thermal decoherence, and its limitations}

Forces between macroscopic objects are often the consequence of interaction with smaller particles and/or excitations in the surrounding medium. For our purpose, it is useful to consider then two parallel plates immersed in a thermal molecular gas that are a distance $a$ apart. One might think that the molecules impinging on the plates from the outside will generate a net pressure pushing them towards each other. This conflicts with basic expectation that in a system with uniform pressure no net force can be exerted on any object. The nice resolution is shown in Fig. 1a showing that the rare 'spoiler' molecules that sneak in between the plates keep moving back and forth and reflecting from the plates exactly counter the effect of all the many outside collisions. If, however, as shown in Fig. 1b, the gas molecules have a distribution of sizes and in particular have diameters of order a, then they will not be able to go inside and a net apparent attraction between the plates will thus be generated.

It seems natural to extend these simplistic arguments—-which indeed have been verified in colloidal systems-to a gas of thermal photons. Indeed photons/e.m modes of wavelengths $\lambda$ exceeding the plate separation $a$-cannot propagate inside this "wave guide'. Such modes cannot serve then as "spoilers" for the inward pressure from the outside collisions. The number density of such modes of wavelength larger than $\lambda \approx a$ is $\approx a^{-3}$. In the $k_{B} T>\hbar c / a$ regime, each mode has an energy of $k_{B} T$ (and each mode is populated by $k_{B} T /(\hbar \omega) \approx k_{B} T a /(\hbar c) \approx \xi$ quanta). The resulting net pressure and attractive force paper unit area $F_{\text {Casimir }}(a, T) / A$ is $\approx T / a^{3}$ as predicted by the path integral formulation above.

It is well known that many phenomena in Q.E.D derive and/or can be attributed to the existence of the vacuum fluctuations with each mode carrying 'half' a quantum or zero point energy of $\hbar \omega / 2$. A simple repetition of the above leads to $F_{\text {Casimir }}(V a c) \approx 1 / a^{4}$. However, when pushed further to more subtle effects such as the dependence of the force on the angle $\beta$ between the directions of conductivity of the two plates which is the topic of interest here, the naive argument seems to completely fail.

To see this, let us consider the following setup which hopefully can be used to heuristically motivate and in some subtle incarnation also measure the effect. Instead of having the parallel Casimir plates made of full metal sheets, we have two frames strung with sufficiently dense and thin parallel wires. These then correspond to the Casimir plates with the preferred conductance directions namely along the wires in each frame. The two extreme cases studied above then correspond to the setting of Figs. 1a and $b$.

In the first case, only photons polarized along the common-say $x$ direction—of the wire will be effectively reflected by the induced currents in the thin wires. We then expect that the Casimir force in such an arrangement will be reduced by a factor half as and amusingly this was indeed found both for the $T=0$ and for the $T \neq 0$ cases by the path integral method. If however the wires are crossed at 90 degrees then the polarized photons coming from the outside will sail through the left mesh and then reflect back from the right mesh. However, since an equal amount of y polarized photons reflect from the outside of the right mesh-no net force should arise. This is flatly negated by the path integral computations.

First, it was found [4] that for the $T=0$ case, the force in the crossed case, Fig. $2 b$ is reduced only by $\approx 1 / 2$ as compared to the parallel wires case, Fig. 2a. This can be 'explained' by the fact that our argument naively assumed that all photons move along the $z$ direction whereas all direction of incidence should be considered. The key question still remains-why did we go so wrong in the thermal case and what makes here the direction/polarization effect decay so fast-as $\exp (-\xi)=\exp \left(-2 \pi k_{B} T a /(\hbar c)\right)$ ?

We believe that the answer is relatively simple though instructive. The naive argument fails because of the decoherence effects at finite temperature. Clearly, the path integral calculation which is far more sophisticated 

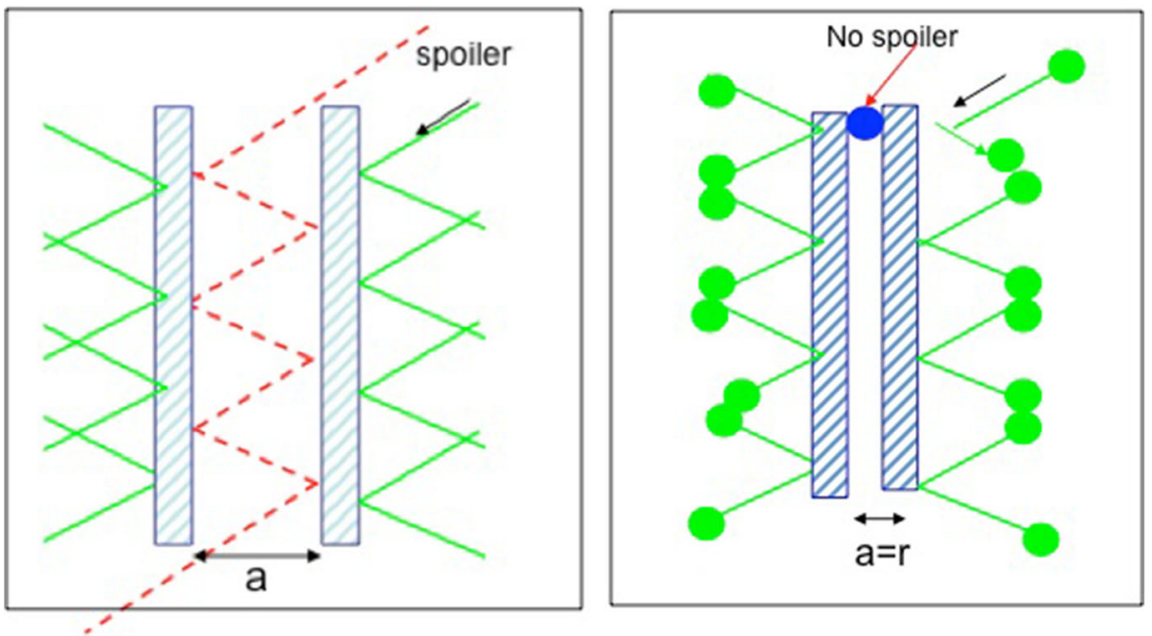

Fig. 1 A cartoon of a classical analog of the Casimir effect. Left a cancelation of outside and internal forces leading to no net attraction between the plates. Right if the gas particles are of a "quantized" minimal size, there are no internal forces and thus an overall attraction between the plates when they are close to each other
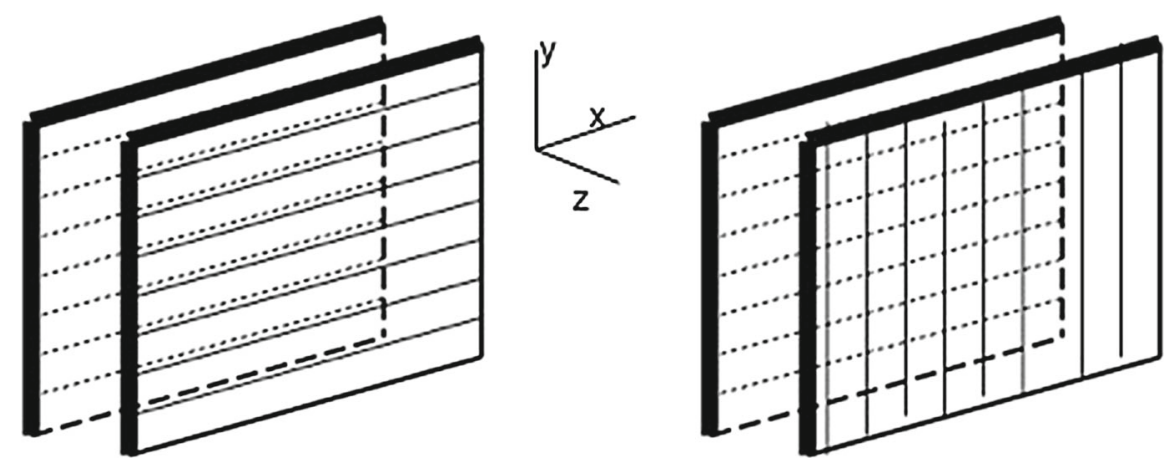

Fig. 2 Plates that conduct along one direction for different angular orientations of the conducting directions. Left parallel plates $(\beta=0)$. Right orthogonal plates $(\beta=\pi / 2)$

seems superior and is indeed correct. Specifically, we expect that practically at all times the photons are not in any specific polarization state but in an equal mixture of both.

The exponential suppression of the effect with temperature could be qualitatively argued for as follows: the average number of photons in any mode $k$ of frequency $\omega$ is $\bar{n}=\left(k_{B} T\right) /(\hbar \omega) \approx \xi$ The probability of having zero thermal photons then is $\exp (-\bar{n})$. Since all thermal photons are maximally mixed, we have then only the ' $1 / 2$ ' vacuum photon contributing the exponentially suppressed effect.

Let us assume that the decoherence time is set just by the temperature and is indeed $t_{\mathrm{dec}}=h /\left(k_{B} T\right)$. If the distance traveled during this time - the decoherence distance $l_{\mathrm{dec}}=\left(c t_{\mathrm{dec}}\right)$ is much larger than the separation of the plates $a$ than while in transit the photon could decohere $\xi=a / l_{d e c}$ times. The probability that it will stay in its original polarization state and contribute to the angular $(\beta)$ dependent force is then reduced by $\exp (-\xi)$.

The key observation is that, up to constants of order unity, $t_{\mathrm{dec}}=h /\left(k_{B} T\right)$ is the shortest minimal decoherence time which is consistent with the quantum uncertainty relation: $\Delta t \Delta E \geq \hbar / 2$ when we use the thermal energy $\left(k_{B} T\right)$ for the energy $\Delta E$. We could however in specific set-ups have it much longer than that this lower bound scale.

It is worth noting that the formal path integral approach in which the attraction between say two charged particles can be viewed as resulting from the joint propagation in the same background field is not completely different from 
what we are discussing albeit in a very simplistic view here. what the naive almost mechanistic approach sorely misses are Aharonov-Bohm (or even Coulomb) phases of the form $\exp \int d x^{\mu} A_{\mu}(x)$.

We next turn to the effects of thermal decoherence on Casimir-like forces which are generated by such AharonovBohm $(\mathrm{AB})$ phases.

\section{Thermal decoherence screens AB phase-induced interactions}

The better known evaluations of the Casimir energy are by summing the energy shifts of all the photon modes in the vacuum, induced by the introduction of the plates at their specified location. It is assumed that the plates or other conducting/ dielectric objects, have large inertia and can be viewed as static. Some time ago it was pointed out [8] that similar "AB type" interactions are generated between magnetic fluxes $\Phi_{1}, \Phi_{2}, \ldots, \Phi_{n_{F}}$ located at given points $R_{1}, \ldots, R_{n_{F}}$ inside a two-dimensional region where the wave function of $\mathrm{N}$ electrons is non-vanishing. The introduction of the fluxes which play here the role of the heavy degrees of freedom modifies the ground state wave function:

$\Psi^{(0)}\left(\mathbf{r}_{1}, \mathbf{r}_{2}, \ldots, \mathbf{r}_{N}\right) \rightarrow \Psi^{(0)}\left(\mathbf{r}_{1}, \mathbf{r}_{2}, \ldots, \mathbf{r}_{N} ; \mathbf{R}_{1}, \Psi_{1}, \mathbf{R}_{2}, \Psi_{2}, \ldots, \mathbf{R}_{n_{F}}, \Phi_{n_{F}}\right)$.

The energy shift relative to the magnetic field-free case, $\delta E^{(0)}$, generated by having magnetic fluxes depends on their sizes and location. This shift generates via a Born Oppenheimer type approximation, an interaction between the fluxes

$\delta E^{(0)}\left(\mathbf{R}_{j}, \Phi_{j}\right)=W\left(\mathbf{R}_{j}, \Phi_{j}\right)$,

where the gradients of $W$ are the forces $\mathbf{F}_{i}$ that act on the fluxes $\Phi_{i}$. In the following, we recall the estimate of this interaction and resulting forces between the fluxons so as to be able to discuss the thermal decoherence-induced screening of theses forces. The calculations are greatly simplified if we first neglect the interaction between the electrons so that the ground state is antisymmetrized product state (filling up a Fermi circle in an appropriate parametrization). The total energy then is simply the sum of the shifts of the individual states

$\delta E^{(0)}\left(\mathbf{R}_{j}, \Phi_{j}\right)=\sum_{i=1}^{N} \delta E_{\gamma_{i}}\left(\mathbf{R}_{j}, \Phi_{j}\right)$.

A sense of the size of the effect is obtained by considering first just one flux $\Phi=\left(\alpha \Phi_{0}\right)$ at the center of a circular disc of radius $R$ with a uniform two-dimensional electron density $n_{2}$. The fluxon $\Phi_{0}=h c / e$ is the flux quantum appropriate to the charge e of a single electron. By rotational symmetry, the initial electron wave functions are eigenstates of the angular momentum $L_{z}$ with integer eigenvalues $l=0, \pm 1, \pm 2, \ldots$. For the purpose of finding the effect of the fluxes, the exact $J_{n}^{l}(k r) \exp i l \phi$ form for the wave functions, can be WKB approximated by semiclassical paths of fixed radii. The introduction of the fluxon increases all of the $l$ values by $\alpha$,

$|l| \rightarrow|l|+\alpha$ for $l \geq 0, \quad|l| \rightarrow|l|-\alpha$ for $l<0$,

and correspondingly modifies the relevant angular parts of the energies $E_{l, r}^{0}=|l|^{2} \hbar^{2} /\left(2 m r^{2}\right)$.

The sum of the energy shifts for a single $l=+,-|l|$ pair is then shifted by $\delta E_{|l|, r}^{(0)}=\frac{\hbar^{2} \alpha^{2}}{2 m r^{2}}$. Summing over all $l$ and $r$ values so as to account for all the $N=n_{2} \pi R^{2}$ electron states, we find that the total energy shift is:

$W_{\alpha}^{\text {tot }}=\sum_{|l|, n} \frac{\hbar^{2} \alpha^{2}}{2 m r_{n}^{2}}, \frac{\alpha^{2}}{2} \frac{n_{2} \hbar^{2}}{2 m} \int_{0}^{R} \frac{2 \pi r d r}{r^{2}}=\frac{\pi}{2} \frac{\alpha^{2} n_{2} \hbar^{2}}{2 m} \ln \left(\frac{R}{a_{0}}\right)$,

where $a_{0}$, the distance between the electrons serves as a lower cutoff.

The logarithmic dependence of $W(R)$ on the size of the system reflects an underlying scaling invariance of the effective two-dimensional potential generated by the fluxes. It suggests that the interaction between two fluxons $\Phi_{1}$ and $\Phi_{2}$ at a distance $a=\left|R_{1}-R_{2}\right| \ll R$ and far from the boundaries is:

$W\left(\alpha_{1}, \alpha_{2}\right)=\xi_{\left(\alpha_{1}, \alpha_{2}\right)} \frac{\pi}{16} \frac{n_{2} \hbar^{2}}{m} \ln \left(\frac{a}{a_{0}}\right)$, 
with $\xi_{\left(\alpha_{1}, \alpha_{2}\right)}$ some function of the individual fluxes and a logarithmic dependence on the relative distance.

Unlike the Casimir force, the present force is of a topological nature. The energy $W_{\alpha}(R)$ due to a fluxon $\alpha$ is proportional to $\alpha^{2}$ only for $\alpha \leq 1 / 2$. Integer $\alpha$ amounts to pure gauge as a shift of all angular momenta by an integer amounts to a negligible "surface" effect modifying the energy of states with angular momenta near $l_{\max }$ only. This and the time reflection symmetry corresponding to $L_{z} \rightarrow-L_{z}$ imply that $W_{\alpha}(R)$ is maximal for $\alpha=1 / 2$ and falls off as $(1-\alpha)^{2}$ in the $[1 / 2,1]$ interval and periodically repeats beyond that. For this reason, the pair-wise force is maximal between fluxes of size $|\alpha|=1 / 2$. Furthermore, the force is attractive even when the latter are parallel to each other since as the fluxons move closer together more electrons see just the effective total and trivial integer flux and pick no phase upon encircling the pair:

$F_{(1 / 2,1 / 2)}(a) \simeq \frac{\xi \pi}{16} \frac{n_{2} \hbar^{2}}{m} \frac{1}{a}$.

The topological nature also manifests in that no force is experienced by a fluxon located outside the region where the electrons are confined since the phases picked up by the electrons when traveling along any closed loop-which drive all these effects,- - do not depend on the location of outside fluxon.

Half-fluxons that are parallel to each other are particularly relevant as they may naturally occur in superconductors. Unfortunately this makes calculations for assessing the observability of this new type of force much harder than for the case of the formally somewhat similar Casimir effect. The Casimir plates are well-defined macroscopic objects whereas the fluxons are generated via a collective effect of the Cooper pairs and electrons in the superconductor, i.e., the basic element comprising the medium which generates the new force between the fluxons. It was conjectured [8] that the large mutual interaction energies of the fluxons might induce some counter-currents of electrons which weaken the other-wise very strong force.

Here, we focus on the more concrete thermal decohering which can reduce the force. It too is connected with the specific material effects. In the case of the Casimir plates, one could reduce the ambient temperature to very low values so that (when all constants are restored) the dimensionless product $\xi=a k_{B} T /(\hbar c)$ is small enough and also the path integral formal calculation will allow a measurable angle-dependent Casimir forces. This however cannot be done here even in principle. The temperature $T$ of the superconductor where we propose to study possible attraction between fluxons must be a finite fraction $f=\mathcal{O}(1)$ of the critical temperature for the onset of superconductivity, $T=f T_{c}$. Only then will a finite fraction $u=F(f)$ of all the electrons remain unpaired into cooper pairs, and only these unpaired electrons generate the force between the half-fluxons.

Thus, we have to address the question of the thermal decoherence effects on the new force between the fluxons. To disentangle this from other matter linked effects, we use the idealization where the fluxons can be viewed as external rigid entities immersed into the electrons. We could then compute the "Casimir like force" per unit length due to the electrons in the medium using a path integral and then include the thermal effects by compactifying the euclidean time direction to the Distance 1/T. Two technical difficulties may hinder such a calculation. First, even for non-interacting electrons, the path integral has the sign problem arising from the Fermi statistics and the nonvanishing chemical potential. Also, the symmetry of the problem is reduced from the $3 \mathrm{D}$ translational invariance (in the $x, y$ and $t$ directions) when we have the parallel infinite Casimir plates geometry to one with only a spatial invariance with $z$ the direction of the fluxons. ${ }^{2}$

We will not attempt such a calculation here. We believe, however, that at $T=0$, it will reveal the logarithmic interaction energy of [8]. Also as in the path integral calculation of the Casimir force, at finite $T$, this long range interaction will be screened and fall exponentially with a characteristic decoherence length $l_{\mathrm{dec}}=l_{\mathrm{scr}}$. It derives from the decoherence time $t_{\mathrm{dec}}=1 / T$ for $T \approx T_{c}$ via $l_{\mathrm{dec}}=t_{\mathrm{dec}} v_{\mathrm{Fermi}}$ as the latter Fermi velocity is the velocity of the electrons. For the force to be readily measurable, we need that the average distance $L$ between the parallel fluxes in an Abrikosov lattice be smaller than $l_{\text {scr. }}$. But $L$ must be greater than the radius $\mathrm{d}$ of the individual fluxons which, in turn, is of the order of the London penetration length $\lambda_{L}$. The condition then becomes $l_{\text {coh }}>\lambda_{L}$, whereas the opposite inequality characterizes the type II superconductors in which Abrikosov flux lattices exist. Indeed, the

2 To see thermal screening it suffices to consider a single fluxon in which case we can use, as done above, the extra rotational symmetry to help facilitate the calculation. 
very existence of such lattices requires that the ordinary magnetic repulsive force between the parallel fluxons will dominate. $^{3}$

\section{Summary and conclusions}

In the current work, we discussed two quantum systems (involving the Casimir and AB type effects) and examined their stability to thermal decoherence.

In the first case, an explicit path integral calculation for the finite temperature system, suggested substantial decoherence for the polarized Casimir force rendering experimental attempts for measuring the relative conductance angle dependence force unlikely to succeed.

We have not been able to perform an explicit $T=0$ and $T \neq 0$ path integral evaluation of the second system with $\mathrm{AB}$ force between fluxons in a superconductor reflecting the phases (sign flip) picked up by unpaired electrons circulating the fluxons in superconductors. However, similar arguments to those used in the Casimir case suggest that the situation may be notably different. The conventional coherence length for electrons $\hbar v /\left(k_{B} T_{c}\right)$ is smaller in generic type II superconductor than the London penetration length which in turn is smaller than the diameter of a single fluxon let alone the distance between two fluxons. At temperatures $T \approx T_{c} / 2$, the formal minimal de-coherence length which translates into an exponential screening of the original long range force at $l_{\mathrm{scr}}=l_{\mathrm{dec}}$ will then dramatically reduce the new force and make its detection rather difficult.

There are many other obstacles of a more technical nature for observing this truly unique and interesting force such as the existence of impurities and the possible pinning of the fluxons on those. Still it is not inconceivable that a dedicated high precision measurements of Abrikosov lattices for disparate superconductors and temperatures in which both the fraction of unpaired electrons and the (de)coherence length and of the magnetic field B (changing the density of fluxons) may reveal the existence of the force. While differing greatly in many details both of the above examples illustrate albeit the very different, effects of decoherence introduced by finite temperature and both serve as concrete actual examples of the much more formal yet general discussions of the effects of strongly and weakly coupled thermal baths on quantum systems.

Acknowledgments We would like to thank Armin Gulian and Michael Levin for insightful and very helpful comments and Ramanath Cowsik for many discussions over the years and in particular a recent one on classical dissipation which inspired the present work. This work was partially supported by NSF DMR-1106293.

\section{References}

1. Kubo, R.: Statistical mechanics: an advanced course with problems and solutions, 7th ed. Elsevier, North-Holland (1990)

2. Nandkishore, R., Huse, D.A.: Many body localization and thermalization in quantum statistical mechanics. arXiv:1404.0686 (2014)

3. Nussinov, S.: New versions of the Casimir Effect. arXiv:Hep-ph/9509228 (1995)

4. Kenneth, O., Nussinov, S.: A new polarized version of the casimir Effect. Phys. Rev. D 63, 121701 (2001)

5. Kenneth, O.: Various aspects of the Casimir effect. PhD thesis, Tel-Aviv University (2002)

6. Milton, K.: The Casimir effect: physical manifestations of zero-point energy. World Scientific, Singapore (2001)

7. Markle, C.: On the orientation dependence of the Casimir force. PhD thesis, Washington University, St. Louis (2013)

8. Aharonov, A., Nussinov, S., Popescu, S., Reznik, B.: Aharonov-Bohm type forces between magnetic fluxons. Phys. Lett. A 231, 299-303 (1997)

9. Aharonov, A., Coleman, S., Goldhaber, A.S., Nussinov, S., Popescu, S., Reznick, B., Rohrlich, D., Vaidman, L.: Aharonov-Bohm and Berry phases for a quantum cloud of charge. Phys. Rev. Lett 73, 918 (1994)

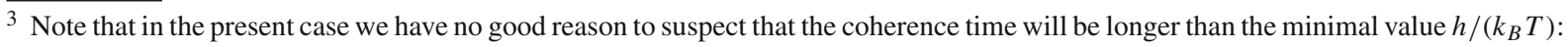
the medium of electrons is dense and we have a very strong electron-electron scattering cross-section. Even upon traveling the short distance say 300 Angstrom between uxons in NbSe2 superconductor an electron is likely to collide with other electrons and randomize its phase.
} 